\title{
Lipid biosynthesis monitored at the single-cell level in Saccharomyces cerevisiae
}

\author{
Pramote Chumnanpuen ${ }^{1}$, Christian Brackmann ${ }^{2}$, Subir K. Nandy ${ }^{1}$, Susana Chatzipapadopoulos ${ }^{2}$, \\ Jens Nielsen ${ }^{1}$ and Annika Enejder ${ }^{2}$ \\ ${ }^{1}$ Systems and Synthetic Biology, Department of Chemical and Biological Engineering, Chalmers University of Technology, \\ Gothenburg, Sweden \\ ${ }^{2}$ Molecular Microscopy, Department of Chemical and Biological Engineering, Chalmers University of Technology, Gothenburg, \\ Sweden
}

There is increasing interest in bioengineering of lipids for use in functional foods, pharmaceuticals, and biofuels. Saccharomyces cerevisiae is a widely utilized cell factory for biotechnological production, thus a tempting alternative. Herein, we show how its neutral lipid accumulation varies throughout metabolic phases under nutritional conditions relevant for large-scale fermentation. Population-averaged metabolic data were correlated with lipid storage at the single-cell level monitored at submicron resolution by label-free coherent anti-Stokes Raman scattering (CARS) microscopy. While lipid droplet sizes are fairly constant, the number of droplets is a dynamic parameter determined by glucose and ethanol levels. The lowest number of lipid droplets is observed in the transition phase between glucose and ethanol fermentation. It is followed by a buildup during the ethanol phase. The surplus of accumulated lipids is then mobilized at concurrent glucose and ethanol starvation in the subsequent stationary phase. Thus, the highest amount of lipids is found in the ethanol phase, which is about $0.3 \mathrm{fL} / \mathrm{cell}$. Our results indicate that the budding yeast, S. cerevisiae, can be used for the biosynthesis of lipids and demonstrate the strength of CARS microscopy for monitoring the dynamics of lipid metabolism at the single-cell level of importance for optimized lipid production.

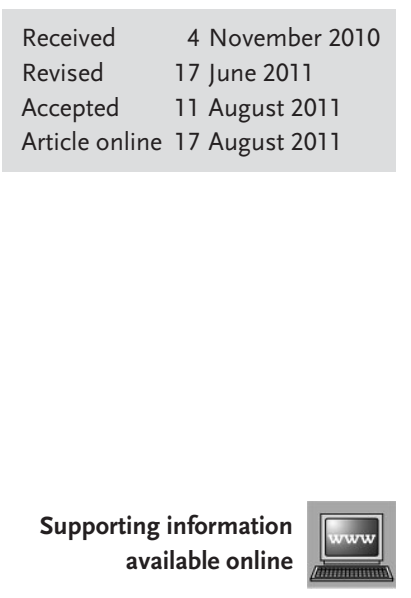

Keywords: CARS microscopy · Lipid droplet quantification · Lipid storage · Nutrient Stress · Saccharomyces cerevisiae

\section{Introduction}

Baker's yeast, Saccharomyces cerevisiae, is an important cell factory used for the biotechnological production of a range of fuels, chemicals, and food ingredients. However, its ability to produce lipid species as alternatives to vegetable oils for human

Correspondence: Prof. Annika Enejder, Molecular Microscopy, Department of Chemical and Biological Engineering, Chalmers University of Technology, Kemivägen 10,

SE-412 96 Gothenburg, Sweden

E-mail: enejder@chalmers.se

Abbreviations: Bodipy, boron dipyrromethene; CARS, coherent anti-Stokes Raman scattering; gDW, gram dry weight; HGLN, high glucose, low nitrogen; LGHN, low glucose, high nitrogen; TAG, triacyglyceride consumption, petroleum-based fuels, or as components in pharmaceuticals has not been explored, to date, because it accumulates only minor amounts of lipids; estimated at less than $15 \%$ of its biomass [1]. Instead, the focus has been on the use of oleaginous yeasts, such as Yarrowia lipolytica, which have a capability to accumulate lipids at levels corresponding to more than $36 \%$ of their biomass [1]. Unfortunately, to achieve these high lipid levels, special cultivation conditions must be ensured and are presently restricted to laboratory-scale growth [2]. The thought of using S. cerevisiae instead as a study organism for lipid biosynthesis is therefore tempting because this organism is tolerant to harsh industrial conditions, is very well characterized, and is already used for large-scale production of different products. 
All eukaryotic cells have a pool of neutral lipids stored as cytoplasmic droplets, which serve as reservoirs of cellular energy and building blocks for membrane lipids, consisting of triacylglyceride (TAG) and steryl esters [3, 4] surrounded by a monolayer of phospholipids and associated proteins [5-7]. In yeast, the lipid droplets consist of about $50 \%$ each of TAG and steryl esters $[6,8,9]$; the total amount of which is, in general, considered to be low relative to the dry cell mass $(<15 \%)$. However, we hypothesize that the amount of neutral lipid storage in yeast is a highly dynamic variable because a unicellular organism, such as yeast, is able to quickly and easily adjust the internal metabolism to new conditions. Indeed, environmental stress and starvation induce increased synthesis and accumulation of neutral lipids [6, 10]. Clearly, fundamental insights into lipid droplet cell biology are of utmost importance for efficient utilization of the yeast lipid metabolism for the biosynthesis of valuable lipid species using $S$. cerevisiae as a cell factory.

To support this development, we have quantified the accumulation of TAG throughout different metabolic stages (i.e., glucose, ethanol, and stationary phases in batch cultivation) characteristic for industrially grown yeast and carried out unique single-cell monitoring of the three-dimensional distribution and amounts of lipids stored in living, unlabeled cells by coherent anti-Stokes Raman scattering (CARS) microscopy. This emerging technique has several advantages over present technology based on fluorescence microscopy, the main disadvantage of which is necessary labeling with fluorescent markers, resulting in a strong dependence on labeling efficiency or expression of fluorescent protein in the structure of interest. Together with dependence on fluorescence yield, which is determined by the local chemical environment, this introduces variations and uncertainties in quantitative analysis, for example, reported for the commonly used lipid stains Nile red and boron dipyrromethene (Bodipy) [11]. Furthermore, by using labeling, the cells are studied in a modified state with possible effects on their properties and for lipids it was shown that the use of alcohols in staining protocols influenced lipid droplet morphology [12]. It is often also of interest to co-localize visualized lipid droplets with other organelles by means of double labeling protocols. However, this possibility is often limited due to the broad fluorescence emission of Nile red or the combined green and red fluorescence identified for Bodipy and recently addressed by Ohsaki et al. [13]. Fluorescence microscopy is also, in many situations, limited by sample photo-bleaching, in particular, when using pro- tein tagging. Nevertheless, intracellular lipid droplets in $S$. cerevisiae have been visualized by fluorescence tagging the protein Erg6 with green fluorescent protein (GFP) [7]. Thus, the drawbacks of established technology make a label-free method for lipid visualization and quantification without these limitations of high interest. CARS microscopy probes intrinsic molecular vibrations, making it a label-free technique that allows livecell studies under native conditions. In addition, it offers the advantages of three-dimensional imaging with high spatial resolution, good sample penetration, and low risk for photo-induced effects and damage. CARS is a laser-induced nonlinear optical four-wave mixing process in which combined excitation and scattering generates blueshifted antiStokes scattered photons in a sample. An enhanced CARS signal is achieved as the applied laser fields are tuned into resonance with a Raman-active molecular vibration, and thereby, target specific species or molecular groups. Compared with spontaneous Raman scattering, which also allows specific imaging probing molecular vibrations, CARS is induced by resonant vibrational excitation and results in coherent, directed signal emission that allows efficient collection. Thus, CARS signals are orders of magnitude higher than those of spontaneous Raman scattering, effectively reducing image acquisition times, clearly favorable for studies in living cells. CARS probing the symmetric stretching vibration of $\mathrm{CH}_{2}$ groups in lipid acyl chains has become an established method for chemically specific imaging of lipids $[14,15]$ and applied in studies on single cells [16], multi-cellular organisms [17], and tissues [18, 19]. Specific imaging of intracellular lipid droplets in S. cerevisiae by CARS microscopy has also been presented [20].

In this study, the levels of lipid accumulation in yeast were investigated for two nutrient stress conditions: (i) high carbon and low nitrogen access and (ii) low carbon and high nitrogen access [21]. The overall yeast metabolism was monitored by biomass measurements and analysis of all key extracellular metabolites, temporal profiles of $\mathrm{OD}_{600}$, dry cell weight, $\mathrm{CO}_{2}$, and chromatography (HPLC) measurements. In addition, TAG analysis was carried out to obtain a population-based average of triacylglyceride contents for comparison with single-cell data obtained from CARS microscopy. This experimental outline allowed us to monitor and quantify lipid accumulation under conditions typical for large-scale industrial growth at both population-averaged and single-cell levels and to identify phases for optimal lipid yield using the conventional and robust yeast strain $S$. cerevisiae. 


\section{Materials and Methods}

\subsection{Yeast strain and cultivation conditions}

Batch cultivations of yeast strain CEN.PK 113-7D MAT $\alpha$ SUC2 MAL2-8C (Scientific Research and Development $\mathrm{GmbH}$, Germany) were set up in duplicate under two different conditions, corresponding to nitrogen and carbon starvation. Aerobic batch cultivations were carried out in well-controlled 1-L bioreactors (DASGIP) with a working volume of $700 \mathrm{~mL}$. The growth medium employed in the cultivations was that specified by Verduyn et al. [22], but the initial concentrations of nitrogen $\left(\left(\mathrm{NH}_{4}\right)_{2} \mathrm{SO}_{4}\right)$ and carbon (glucose) sources were adjusted, according to the stoichiometry for cell growth as described by Stephanopoulos et al. [23] and the "calorie restriction condition" defined by Lin and Sinclair [24]. A glucose concentration of at least $20 \mathrm{~g} / \mathrm{L}$ and an ammonium sulfate concentration of $3.5 \mathrm{~g} / \mathrm{L}$ are required in the medium to reach a biomass concentration of 12 gram dry weight $(\mathrm{gDW}) / \mathrm{L}$ for the CEN.PK 113-7D yeast strain. Therefore, glucose and $\left(\mathrm{NH}_{4}\right)_{2} \mathrm{SO}_{4}$ concentrations of $20 \mathrm{~g} / \mathrm{L}$ and $2 \mathrm{~g} / \mathrm{L}$, respectively, were used in the medium to achieve a condition of nitrogen starvation, the high glucose, low nitrogen (HGLN) condition. To instead induce a glucose starvation condition (low glucose, high nitrogen (LGHN)), a glucose concentration of $5 \mathrm{~g} / \mathrm{L}$ and a $\left(\mathrm{NH}_{4}\right)_{2} \mathrm{SO}_{4}$ concentration of $3.5 \mathrm{~g} / \mathrm{L}$ were used.

Cells were transferred from the pre-culture to obtain initial cell concentrations corresponding to an $\mathrm{OD}_{600}$ of around 0.01 . The fermentations were carried out with aeration using atmospheric air set to $30 \mathrm{~L} / \mathrm{h}\left(1 \mathrm{~atm} ., 30^{\circ} \mathrm{C}\right)$ and agitation at $800 \mathrm{rpm}$. The temperature was kept constant at $30^{\circ} \mathrm{C}$ and the $\mathrm{pH}$ was maintained at 5.0 by the addition of $2 \mathrm{M}$ $\mathrm{KOH}$. The concentrations of carbon dioxide and oxygen in the exhaust gas were monitored by an off-gas analyzer (DASGIP).

Samples were harvested from the cultivation media every second hour and immediately filtered through a $0.45 \mu \mathrm{m}$ pore-size cellulose acetate filter (VWR) and stored at $-20^{\circ} \mathrm{C}$ before analysis. Biomass production was evaluated by measurements of $\mathrm{OD}_{600}$ and dry cell weight. Glucose, glycerol, ethanol, and acetate concentrations were determined by HPLC analysis using an Aminex HPX87H column (Biorad, Hercules, CA) [25].

\subsection{CARS microscopy}

CARS microscopy measurements were carried out using a laser system consisting of a $\mathrm{Nd}$ :Vanadate pump laser (Picotrain, HighQ Lasers $\mathrm{GmbH}$ ) combined with a ring-cavity optical parametric oscilla- tor (OPO; Levante, APE GmbH). The system provided two laser beams of picosecond pulse trains required to induce the CARS process: one from the $\mathrm{Nd}$ :Vanadate laser at $1064 \mathrm{~nm}$ and one from the $\mathrm{OPO}$ at $817 \mathrm{~nm}$. The frequency shift between the beams corresponds to the symmetric stretching vibration of acyl-chain $\mathrm{CH}_{2}$ groups, at $2845 \mathrm{~cm}^{-1}$, and therefore, gives rise to a resonantly enhanced CARS signal from $\mathrm{CH}_{2}$ bonds in the sample. The copropagating beams were directed into an inverted laser-scanning microscope (Eclipse TE-2000-E, Nikon) equipped with a beam scanner (Nikon C1) and focused on the sample (objective 40x Nikon Plan Fluor, N.A. 1.3). Typical average laser powers at the sample were $20 \mathrm{~mW}$ per beam. The forwardpropagating CARS signal, generated at $663 \mathrm{~nm}$, was collected to a photomultiplier tube (Hamamatsu R6357) equipped with bandpass filters. Three-dimensional image data, $z$ stacks, were acquired by scanning different horizontal planes in the sample. A further description of the CARS microscopy setup can be found in reference [15].

The yeast-cell samples for CARS microscopy were prepared by taking cell culture suspension from the fermentation vessel after 12,21 , and $38 \mathrm{~h}$ of growth. A small volume, $3 \mu \mathrm{L}$, was transferred to a chamber consisting of two cover-glass slides and a gasket (Invitrogen, Secure Seal Spacer $d=9 \mathrm{~mm}$ $h=0.12 \mathrm{~mm}$ ) mounted in a sandwich construction. The cells were immobilized during measurements by coating the lower cover slide with poly-L-lysine to attach the cells to the glass surface.

For each condition and time point studied, $z$ stacks of CARS images were measured, covering an area of $20 \times 20 \mu^{2}(256 \times 256$ pixels $)$ and a depth of $10 \mu \mathrm{m}$ (vertical step size $0.5 \mu \mathrm{m}$ ). The spatial resolution of the CARS microscopy setup was estimated to $0.4 \mu \mathrm{m}$ laterally and $1 \mu \mathrm{m}$ axially, corresponding to a probe volume of $0.13{\mu \mathrm{m}^{3}}$ (fL). Numbers and sizes of lipid droplets in 232 and 197 yeast cells were evaluated for the HGLN and LGHN conditions, respectively.

Image analysis consisted of two major steps: a local intensity thresholding procedure [26] was employed to identify lipid droplets, which were then automatically counted and measured using a plug-in tool (3D Object Counter) for the ImageJ image analysis software [27]. Although the lipid droplets were monitored in three dimensions through the cells, evaluation of droplet size was based on two-dimensional information due to the lower spatial resolution of the microscope in the axial $(z)$ direction. Hence, lipid droplet sizes were determined using the built-in ImageJ particle analyzer routine for analysis of the image plane corresponding to the central position of the imaged cells. 


\subsection{Lipid extraction and TAG measurement}

The freeze-dried samples from each time point were extracted and analyzed in technical replicates. The lipid extraction method was adapted from Bligh and Dyer [28]. $20 \mathrm{mg}$ of freeze-dried yeast cells were incubated with $7 \mathrm{~mL}$ chloroform/methanol $(2: 1, \mathrm{v} / \mathrm{v})$ for $3 \mathrm{~h}$ at $4^{\circ} \mathrm{C}$ on a rotary shaker with a speed of $200 \mathrm{rpm}$ and supplemented with $1.7 \mathrm{~mL}$ of $0.73 \%$ sodium chloride, vigorously shaken. Following centrifugation at $3000 \mathrm{rpm}$ at $4^{\circ} \mathrm{C}$ for $4 \mathrm{~min}$, the organic phase was collected for later analysis, whereas the remaining aqueous phase was vigorously mixed and centrifuged with $5 \mathrm{~mL}$ chloroform/methanol $(85: 15, \mathrm{v} / \mathrm{v})$. The total procedure was repeated three times. The collected organic material was combined and evaporated under a nitrogen stream at $40^{\circ} \mathrm{C}$. Total lipids were dissolved in $0.5 \mathrm{~mL}$ chloroform/methanol $(2: 1, \mathrm{v} / \mathrm{v})$ and kept at $-30^{\circ} \mathrm{C}$. The resulting lipid extract was further fractioned using TLC, in which the lipid solution was applied to a TLC plate (Silica gel 60, $20 \times 20 \mathrm{~cm}, 0.5 \mathrm{~mm}$ layer, Merck, Darmstadt, Germany) using a solvent system of heptane/2-propanol/acetic acid (95:5:1, v/v/v). Individual lipids were visualized by spraying the sample with $0.2 \%$ $2^{\prime}, 7^{\prime}$-dichlorofluoresceine in ethanol and identified by comparison of their $R_{\mathrm{f}}$ values with known standards. The bands corresponding to triacylglycerides were manually scraped off for TAG measurement, using the commercial kit "Triglycerides Liquid Stable Reagent" (Thermo Scientific). Results were given in $\mathrm{mmol} / \mathrm{gDW}$, which was converted into \% (w/w) using an approximate molar mass for TAG in yeast of $819 \mathrm{~g} / \mathrm{mol}$, assuming a composition of 33\% 16-carbon chains (three chains; $769.2 \mathrm{~g} /$ $\mathrm{mol}$ ) and 61\% 18-carbon chains (three chains; $846 \mathrm{~g} /$ mol) [29].

\subsection{Statistical analysis}

To ensure that the biological duplicates in each condition and also the technical replicates were not significantly different, the student paired $t$ test was performed. SPSS software version 17.0 (SPSS Inc, Chicago, IL) was used for all analyses and $P$ values of $\leq 0.05$ were considered to differ significantly.

\section{Results and discussion}

The population-averaged data show general trends for the impact of starvation on the cell metabolism. Under HGLN conditions, the cells took approximately $21 \mathrm{~h}$ to finish their exponential phase with a glucose consumption rate of $0.019 \mathrm{~g} /(\mathrm{gDW} \mathrm{h})$

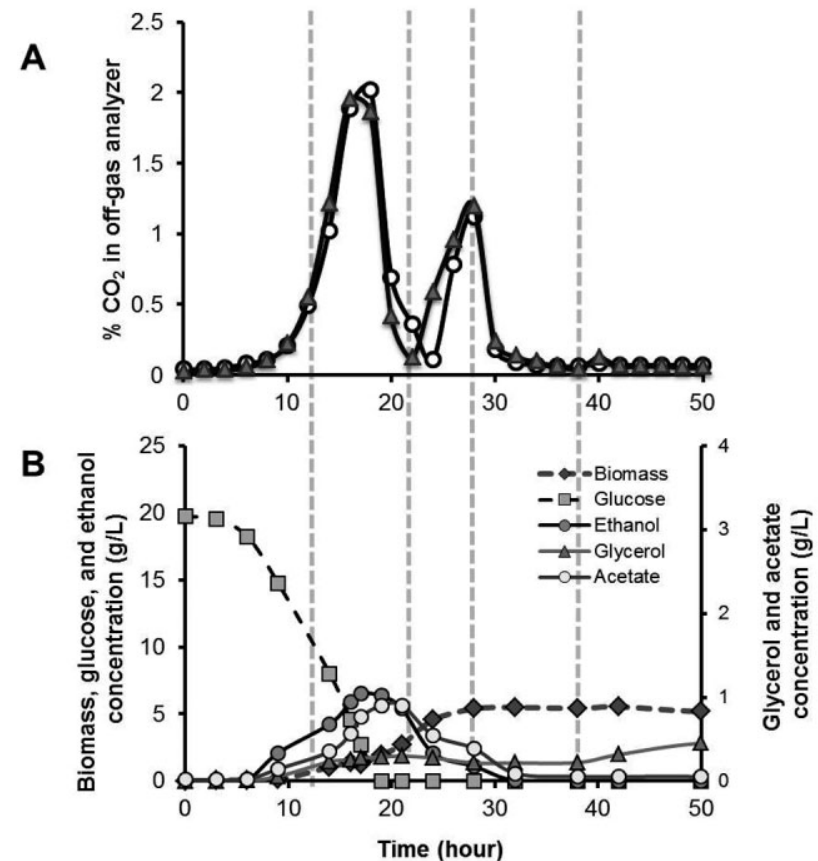

C

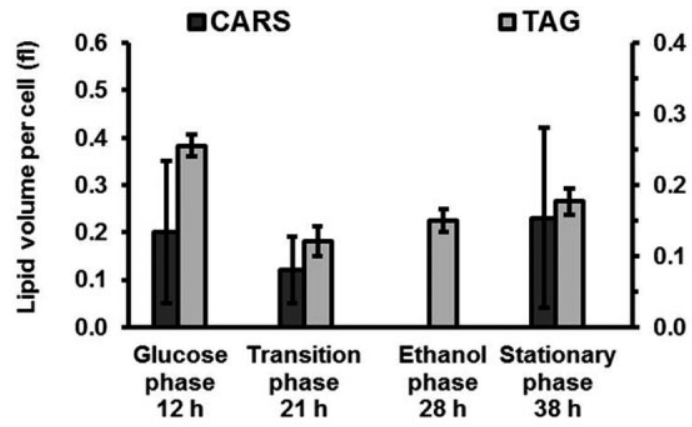

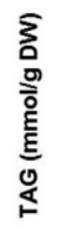

Figure 1. Fermentation profiles for the $\mathrm{HGLN}$ condition. (A) $\mathrm{CO}_{2}$ profiles measured on duplicate fermenters and showing two peaks, representing growth on glucose and ethanol, respectively. The vertical dashed lines represent the time points for lipid analysis (12, 21, 28, and $38 \mathrm{~h}$ ). (B) Profiles of biomass and metabolite concentrations (glucose, glycerol, acetate, and ethanol) measured during fermentation. (C) Cellular lipid content during fermentation presented as average lipid volume per cell with standard deviation (for details, see the Results and discussion), evaluated from CARS microscopy images $(n=232)$ and amount of TAG $(n=6)$. Data are presented as means \pm SD. The evaluated lipid volumes correspond to 4.5 , 3.5 , and 3.8 lipid droplets per cell for the time points 12,21 , and $38 \mathrm{~h}$, respectively.

(Figs. 1A and B and Table 1). In contrast, the condition with glucose starvation and excess nitrogen (LGHN) made the cells finish their exponential phase faster; the shift from glucose to ethanol phase occurred within $15 \mathrm{~h}$ with a glucose consumption rate of $0.012 \mathrm{~g} /(\mathrm{gDW}$ h) (Figs. $2 \mathrm{~A}$ and $\mathrm{B}$, and Table 1). This resulted in 2.5 times less biomass production and a lower maximum specific growth rate than that of the HGLN condition. In addition, the total yields of ethanol, glycerol, and acetate 
Table 1. Specific rates and yields for the two sets of fermentations

\begin{tabular}{|c|c|c|c|c|c|c|}
\hline Condition & $\mu_{\max }^{\text {a) }}$ & $Y_{\mathrm{sx}}^{\mathrm{b})}$ & $Y_{\mathrm{se}}^{\mathrm{c}}$ & $Y_{\text {sg }}^{\text {d) }}$ & $Y_{\text {sa }}^{\mathrm{e})}$ & $r_{s}^{f)}$ \\
\hline HGLN & 0.21 & 0.089 & 0.42 & 0.078 & 0.093 & 0.019 \\
\hline LGHN & 0.17 & 0.070 & 0.35 & 0.035 & 0.046 & 0.012 \\
\hline
\end{tabular}

a) The specific growth rate of biomass (unit: $h^{-1}$ ).

b) Biomass yield on glucose (unit: $g$ biomass formed/g glucose consumed).

c) Ethanol yield on glucose (unit: g ethanol formed/g glucose consumed).

d) Glycerol yield on glucose (unit: g glycerol formed/g glucose consumed).

e) Acetate yield on glucose (unit: g acetate formed/g glucose consumed).

f) Specific glucose uptake rate (unit: g glucose/g biomass/h).

were lower (Table 1) due to a lack of the primary nutrient source, glucose.

Both nutritional conditions (Figs. 1 and 2) showed fermentative metabolism in which consumption of glucose resulted in production of biomass, ethanol, glycerol, and acetate. In parallel, high glucose concentrations lead to a higher production of fatty acids, resulting in an accumulation of lipid droplets, probably by the integration of signals through the protein kinase Snf1 [30]. After $12 \mathrm{~h}$ of growth in the glucose phase, the amount of TAG, about $0.25 \mathrm{mmol} / \mathrm{gDW}(20.5 \% \mathrm{w} / \mathrm{w})$, was similar for both excess and limited access to glucose. The corresponding data obtained from the singlecell CARS microscopy studies were 4.5 and 3.8 lipid droplets per cell on average and evaluated lipid droplet diameters of 0.4 and $0.5 \mu \mathrm{m}$ for the HGLN and LGHN conditions, respectively. Sizes of intracellular lipid droplets in S. cerevisiae were determined, using electron microscopy, by Czabany et al. [31], resulting in diameters in the range of 0.3$0.5 \mu \mathrm{m}$. Typical lipid droplet sizes are thus similar to the spatial resolution of optical microscopy and imaging results in a size overestimation due to convolution with the microscope point-spread function [32]. For CARS microscopy, the nonlinear dependence on excitation intensity and density of probed molecular bonds reduces such an effect to some extent [26]. Nevertheless, an overestimation can still be expected for the diameters evaluated from CARS microscopy, which also seems probable in relation to the electron microscopy results. Assuming spherical lipid droplets, average lipid volumes per cell of $0.2 \mathrm{fL}$ (Figs. 1C and 2C) can be estimated for both conditions and, in relation to the spatial resolution, apparent differences in diameters and calculated volumes must be considered of low significance. Nevertheless, the evaluated averages provide volume estimations as presented in Figs. 1C and 2C. Thus, both methods for lipid quantification indicated that lipid accumulation could not be significantly enhanced by loading the medium with excess amounts of glucose. After $21 \mathrm{~h}$, corresponding to the glucose-ethanol transitional

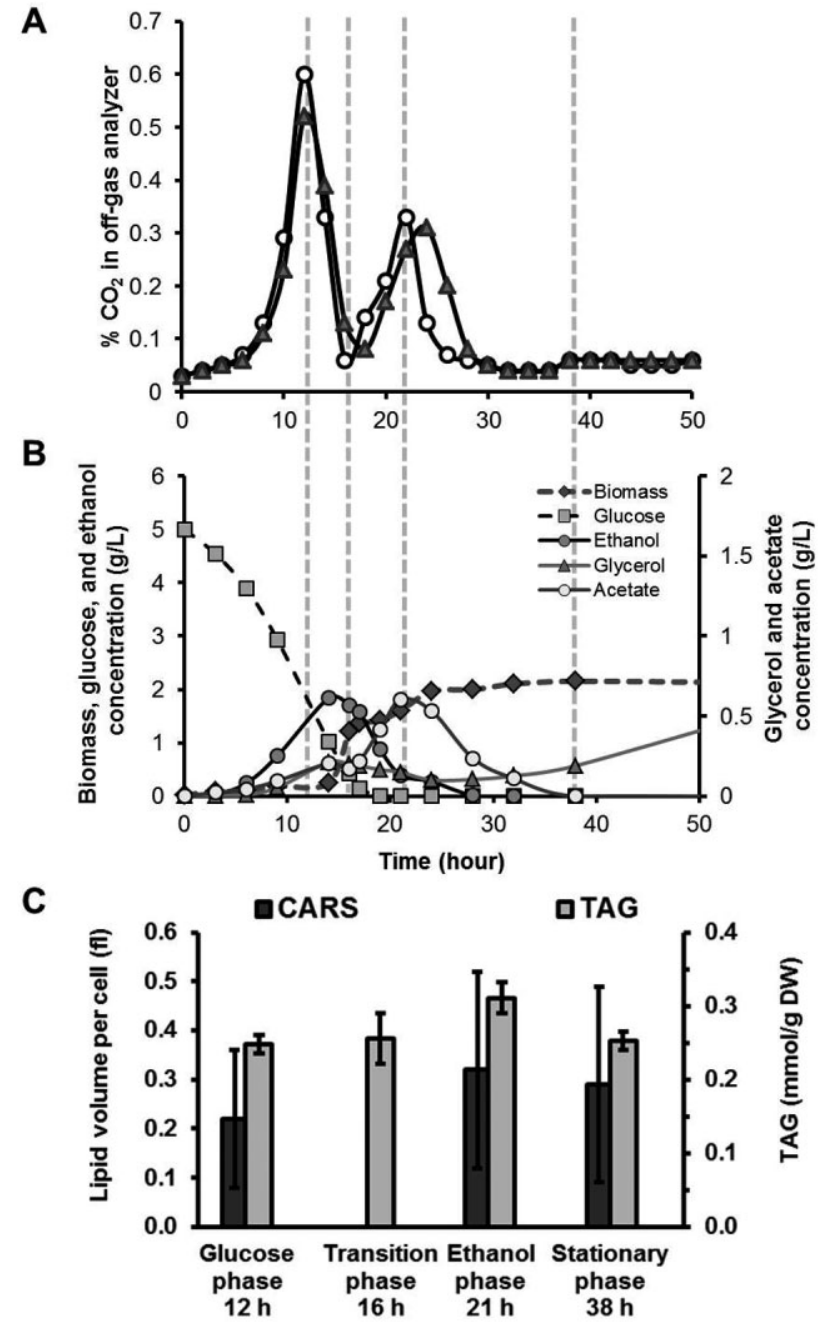

Figure 2. Fermentation profiles for the $\mathrm{LGHN}$ condition (A) $\mathrm{CO}_{2}$ profiles measured on duplicate fermenters and showing two peaks representing growth on glucose and ethanol, respectively. The vertical dashed lines represent the time points for lipid analysis $(12,16,21$, and $38 \mathrm{~h})$. (B) Profiles of biomass and metabolite concentrations (glucose, glycerol, acetate, and ethanol) measured during fermentation. (C) Cellular lipid content during fermentation presented as average lipid volume per cell with standard deviation (for details, see the Results and discussion), evaluated from CARS microscopy images $(n=197)$ and amount of TAC $(n=6)$. Data are presented as means \pm SD. The evaluated lipid volumes correspond to 3.8 , 6.2 , and 3.6 lipid droplets per cell for the time points 12,21 , and $38 \mathrm{~h}$, respectively.

phase for HGLN fermentation, the TAG amount decreased to around $0.12 \mathrm{mmol} / \mathrm{gDW}(9.8 \% \mathrm{w} / \mathrm{w})$, also found by CARS measurements, corresponding to 3.5 lipid droplets per cell on average. With an evaluated droplet diameter of $0.4 \mu \mathrm{m}$, this amounts to a lipid volume of $0.1 \mathrm{fL}$ per cell for this condition and time point. The observed decrease is reasonable, considering that the cells need to mobilize TAG when glucose is depleted before ethanol metabolism increases and becomes effective. In con- 
trast, the amount of TAG remained constant for LGHN conditions during the glucose-ethanol transition phase at $16 \mathrm{~h}$ (Fig. 2C). For the ethanol phase, an increase in TAG to about $0.31 \mathrm{mmol} / \mathrm{gDW}(25.4 \%$ $\mathrm{w} / \mathrm{w})$ and an increase in the number of lipid droplets to 6.2 per cell (diameter $0.5 \mu \mathrm{m}$, volume per cell $0.3 \mathrm{fL}$ ) is observed (Fig. 2C). A slight increase in TAG level $(\approx 20 \%)$ was found after $28 \mathrm{~h}$ when the cells shifted to the ethanol phase in HGLN cultivation (Fig. 1C). Under the LGHN fermentation conditions, lipid accumulation is stimu- lated by conversion of ethanol into lipid precursors in S. cerevisiae [33], exhibiting TAG densities also reported for the oleaginous yeast strain Yarrowia lipolytica [1]. The quantitative single-cell analysis made by CARS microscopy indicates that by harvesting the population in the ethanol phase, when many cells show a high number of lipid droplets, the maximal lipid yield can be achieved, estimated to be $0.3 \mathrm{fL}$ per cell. In the subsequent stationary phase, sampled at $38 \mathrm{~h}$, both conditions show similar numbers of lipid droplets per cell: 3.8 and 3.6 for $x-y$
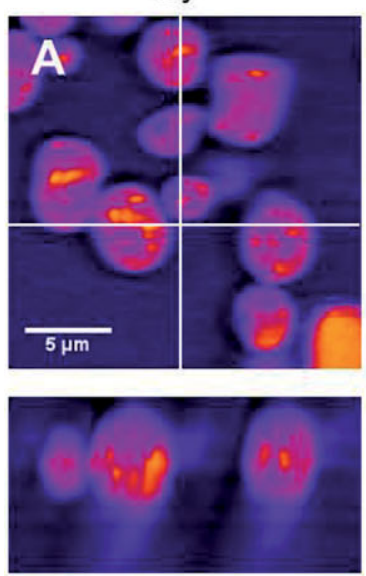

$\mathbf{x - z}$

$x-y$
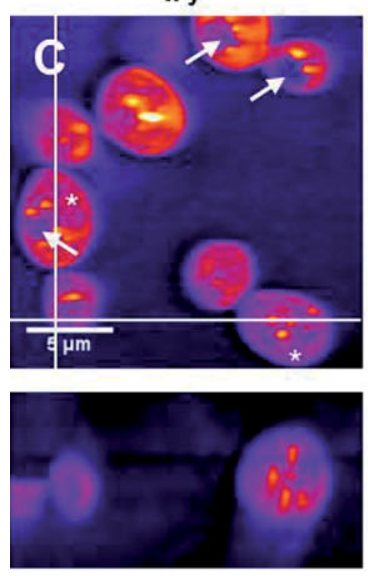

$\mathbf{x - z}$

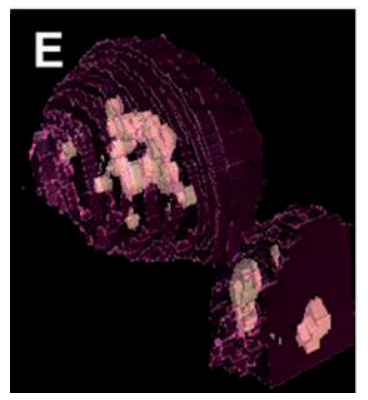

$\mathbf{y - z}$

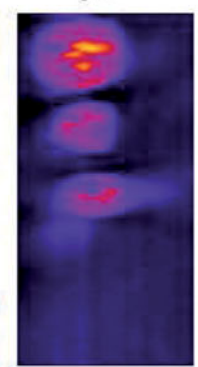

$\mathbf{y - z}$
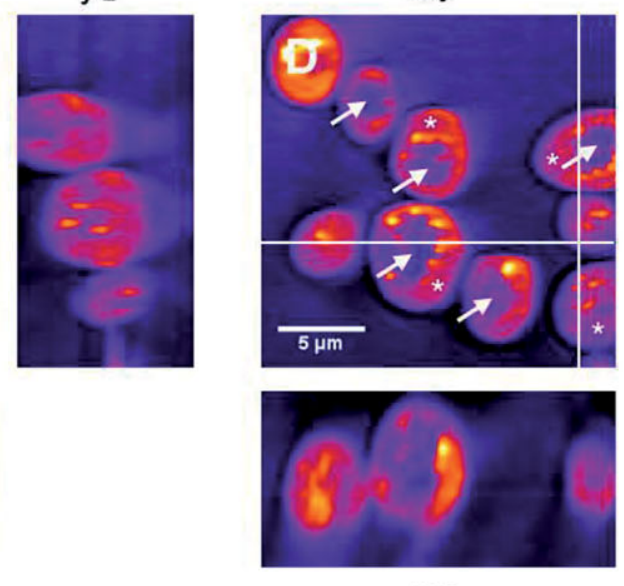

$\mathbf{x - z}$

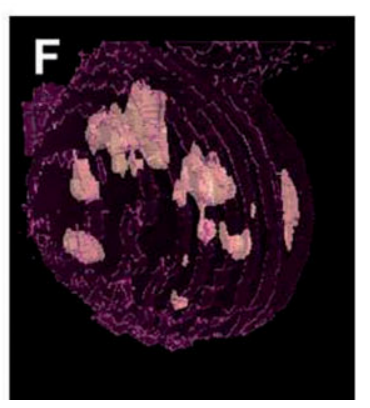

$y-z$

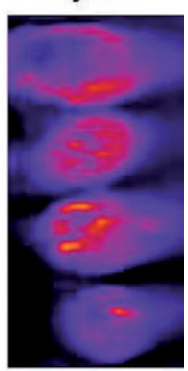

$\mathbf{y - z}$

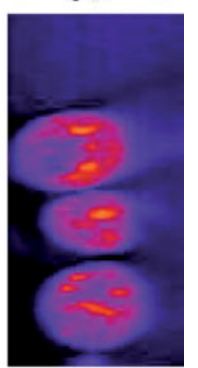

Figure 3. CARS microscopy images showing horizontal $(x-\gamma)$ and vertical $(x-z, y-z)$ views of yeast cells measured after growth for $21 \mathrm{~h}$. Positions of the vertical views are indicated by white lines. Image sizes, $20 \times 20 \mu \mathrm{m}$ and $20 \times 10 \mu \mathrm{m}$. Cells grown under HGLN conditions ( $\mathbf{A}$ and $\mathbf{B}$ ), on average show a lower number of lipid droplets per cell compared with cells exposed to LGHN conditions (C and D). The higher average lipid content for the LGHN condition is represented by cells containing multiple lipid droplets indicated by an asterisk in (C) and (D). The two conditions also show different lipid droplet distribution patterns. Whereas HGLN cells in the transition phase exhibit an even distribution of lipid droplets throughout the intracellular volume, LGHN cells enter the ethanol phase and lipid droplets can often be observed in the periphery surrounding larger vacuoles (indicated by arrows). This arrangement can also be seen in the rendered volume images, showing complete three-dimensional representations of yeast cells (magenta) and lipid droplets (yellow) under HGLN (E) and LGHN (F) conditions. 
HGLN and LGHN, respectively. The evaluated average lipid droplet diameters were $0.5 \mu \mathrm{m}$ for both HGLN and LGHN conditions at this time point and calculated volumes per cell were 0.2 and $0.3 \mathrm{fL}$, respectively. A difference could be observed in the corresponding TAG levels, $0.18 \mathrm{mmol} / \mathrm{gDW}(13.86 \%$ $\mathrm{w} / \mathrm{w})$ for HGLN conditions and $0.25 \mathrm{mmol} / \mathrm{gDW}$ $(19.25 \% \mathrm{w} / \mathrm{w})$ for LGHN condition. Thus, in the stationary phase, TAG production increased following the ethanol phase for HGLN, whereas TAG was mobilized for LGHN. CARS microscopy measurements signify that the mechanisms of droplet formation appear to be a fundamental, standardized process in which average sizes and lipid densities are more or less constant, irrespective of nutritional conditions or fermentative phases. Note that the normalized CARS signal varied by merely $15 \%$ throughout the different metabolic conditions investigated, indicating similar lipid droplet densities.

The error bars for the evaluated volumes per cell (Figs. 1C and 2C) indicate cell-to-cell variations also observed in the CARS microscopy images shown in Figs. 3A-D. Images were measured after growth for $21 \mathrm{~h}$ for the HGLN (Figs. 3A and B) and LGHN (Figs. 3C and 3D) conditions. In addition to horizontal $x-y$ plane views, vertical $x-z$ and $y-z$ plane views measured at the positions of the white lines are shown. Even though individual HGLN cells in Figs. 3A and B show up to 5 droplets per cell, most cells show less, resulting in the reported average value of 3.5 droplets per cell for this condition and time point. In contrast, a higher number of lipid droplets per cell was more common for LGHN (Figs. 3C and 3D, cells indicated by asterisks). CARS data show a larger spread than the TAG levels, based on measurements of much larger cell populations. However, it should be noted that the data spread does not represent the precision of the CARS technique, but rather cell-to-cell variations encountered with any microscopy technique based on single-cell measurements. Nevertheless, it is interesting to see that the average trends of the CARS measurements are consistent with those of the TAG levels. More in-depth comparison between data evaluated from CARS microscopy images and TAG values needs to take lipid droplet sizes more into account and is dependent on the previously discussed size accuracy. In addition, effects such as cell shrinking, resulting in a reduction in cell size without a decrease in cell mass, may need to be considered for a comparison of CARS and TAG results of the stationary phase.

In addition to quantitative measurements of lipid content at the single-cell level, CARS microscopy $z$ stacks provide unique three-dimension- al information on the lipid droplet distribution represented by the planar horizontal and vertical views shown in Figs. 3A-D and the volume images in Figs. 3E and F. This allows us to evaluate the intracellular arrangement of the lipid droplets and a significant difference was observed for the two nutritional conditions after $21 \mathrm{~h}$. Whereas the lipid droplets were evenly distributed in cells under HGLN conditions (still in the transition phase at $21 \mathrm{~h}$ ), they were located in the periphery of cells exposed to LGHN conditions (early ethanol phase at $21 \mathrm{~h}$ ). This was most likely due to the formation of vacuoles in many LGHN cells, of which $89 \%$ exhibited a vacuole, in contrast to the HGLN cells (18\%). This trend can be seen in Fig. 3, where several of the LGHN cells in Figs. 3C and D have a vacuole, as indicated by arrows. This difference in lipid droplet arrangement is also shown in the single-cell volume images in Figs. $3 \mathrm{E}$ and $\mathrm{F}$ (see the Supporting information for $360^{\circ}$ rendering movies).

Together, the results clearly show that there is dynamic regulation of the lipid metabolism in the widely utilized yeast strain $S$. cerevisiae and these dynamic changes can be captured at the single-cell level using CARS microscopy. The accumulation of TAG involves both nutrient sensing of nitrogen, carbon, and energy sources [6], probably by the integration of signals through the protein kinase Snf1 [30].

\section{Concluding remarks}

We have demonstrated the strength of combining population-averaged metabolic analysis with single-cell imaging using CARS microscopy. S. cerevisiae, which is widely used in the biotechnical industry, had a highly dynamic ability to accumulate lipids, depending on nutritional conditions and fermentative phase. By controlling parameters such as nitrogen, carbon, and energy sources, lipid biosynthesis can be influenced. Interestingly, the amount of accumulated lipids during the glucose phase was not significantly influenced by the initial glucose concentration, indicating that it could not be used to enhance lipid synthesis. The highest amounts of accumulated neutral lipids, about $0.3 \mathrm{fL} / \mathrm{cell}$ and $0.31 \mathrm{mmol} \mathrm{TAG} / \mathrm{gDW}(25.4 \% \mathrm{w} / \mathrm{w})$, were found in the ethanol phase and exceeded the amount of lipids synthesized during glucose fermentation.

Part of this work was financed by the Chalmers Foundation and the Knut and Alice Wallenberg Foundation. We also acknowledge financial support from 
the EU-funded project UNICELLSYS, the Swedish Scientific Research Council, and the European Cooperation in Science and Technology (COST). P.C. would also like to thank the Office of the Higher Education Commission, Thailand for support by grant under the Strategic Scholarships for Frontier Research Network for Ph.D. program.

The authors have declared no conflict of interest.

\section{References}

[1] Beopoulos, A., Cescut, J., Haddouche, R., Uribelarrea, J. L. et al., Yarrowia lipolytica as a model for bio-oil production. Prog. Lipid Res. 2009, 48, 375-387.

[2] Bankar, A. V., Kumar, A. R., Zinjarde, S. S., Environmental and industrial applications of Yarrowia lipolytica. Appl. Microbiol. Biotechnol. 2009, 84, 847-865.

[3] Goodman, J. M., Demonstrated and inferred metabolism associated with cytosolic lipid droplets. J. Lipid Res. 2009, 50, 2148-2156.

[4] Zweytick, D., Athenstaedt, K., Daum, G., Intracellular lipid particles of eukaryotic cells. Biochim. Biophys. Acta 2000, 1469, 101-120.

[5] Brown, D. A., Lipid droplets: Proteins floating on a pool of fat. Curr. Biol. 2001, 11, R446-449.

[6] Mullner, H., Daum, G., Dynamics of neutral lipid storage in yeast. Acta Biochim. Pol. 2004, 51, 323-347.

[7] Natter, K., Leitner, P., Faschinger, A., Wolinski, H. et al., The spatial organization of lipid synthesis in the yeast Saccharomyces cerevisiae derived from large scale green fluorescent protein tagging and high resolution microscopy. Mol. Cell. Proteomics 2005, 4, 662-672.

[8] Leber, R., Zinser, E., Zellnig, G., Paltauf, F., Daum, G., Characterization of lipid particles of the yeast, Saccharomyces cerevisiae. Yeast 1994, 10, 1421-1428.

[9] Schaffner, G., Matile, P., Structure and composition of bakers-yeast lipid globules. Biochem. Physiol. Pflanz. 1981, 176, 659-666.

[10] Gasch, A. P., Werner-Washburne, M., The genomics of yeast responses to environmental stress and starvation. Funct. Integr. Genomics 2002, 2, 181-192.

[11] Gocze, P. M., Freeman, D. A., Factors underlying the variability of lipid droplet fluorescence in MA-10 Leydig tumor cells. Cytometry 1994, 17, 151-158.

[12] Fukumoto, S., Fujimoto, T., Deformation of lipid droplets in fixed samples. Histochem. Cell Biol. 2002, 118, 423-428.

[13] Ohsaki, Y., Shinohara, Y., Suzuki, M., Fujimoto, T., A pitfall in using BODIPY dyes to label lipid droplets for fluorescence microscopy. Histochem. Cell Biol. 2010, 133, 477-480.

[14] Le, T. T., Yue, S., Cheng, J. X., Shedding new light on lipid biology with coherent anti-Stokes Raman scattering microscopy. J. Lipid Res. 2010, 51, 3091-3102.

[15] Enejder, A., Brackmann, C., Svedberg, F., Coherent antiStokes Raman scattering microscopy of cellular lipid storage. IEEE J. Sel. Top. Quantum Electron. 2010, 16, 506-515.

[16] Nan, X., Cheng, J. X., Xie, X. S., Vibrational imaging of lipid droplets in live fibroblast cells with coherent anti-Stokes Raman scattering microscopy. J. Lipid Res. 2003, 44, 22022208.
[17] Hellerer, T., Axang, C., Brackmann, C., Hillertz, P. et al., Monitoring of lipid storage in Caenorhabditis elegans using coherent anti-Stokes Raman scattering (CARS) microscopy. Proc. Natl. Acad. Sci. USA 2007, 104, 14 658-14 663.

[18] Huff, T. B., Shi, Y., Fu, Y., Wang, H., Cheng, J. X., Multimodal nonlinear optical microscopy and applications to central nervous system imaging. IEEE J. Sel. Top. Quantum Electron. $2008,14,4-9$.

[19] Le, T. T., Langohr, I. M., Locker, M. J., Sturek, M., Cheng, J. X., Label-free molecular imaging of atherosclerotic lesions using multimodal nonlinear optical microscopy. J. Biomed. Opt. 2007, 12, 054007.

[20] Brackmann, C., Norbeck, J., Åkeson, M., Bosch, D. et al., CARS microscopy of lipid stores in yeast: the impact of nutritional state and genetic background. J. Raman Spectro. 2009, 40, 748-756.

[21] Nandy, S. K., Venkatesh, K. V., Effect of carbon and nitrogen on the cannibalistic behavior of Bacillus subtilis. Appl. Biochem. Biotechnol. 2008, 151, 424-432.

[22] Verduyn, C., Postma, E., Scheffers, W. A., Vandijken, J. P., Effect of benzoic acid on metabolic fluxes in yeasts - a continuous-culture study on the regulation of respiration and alcoholic fermentation. Yeast 1992, 8, 501-517.

[23] Stephanopoulos, G. N., Aristidou, A. A., Nielsen, J., Metabolic Engineering: Principles and Methodologies, Academic Press, San Diego, 1998, pp. 119-121.

[24] Lin, S. J., Sinclair, D., Molecular mechanisms of aging: Insights from budding yeast, in: Guarente, L. P., Partridge, L., Wallace, D. C. (Eds.), Molecular Biology of Aging, Cold Spring Harbor Laboratory Press, Cold Spring Harbor, 2008, pp. 483-516.

[25] Zaldivar, J., Borges, A., Johansson, B., Smits, H. P. et al., Fermentation performance and intracellular metabolite patterns in laboratory and industrial xylose-fermenting Saccharomyces cerevisiae. Appl. Microbiol. Biotechnol. 2002, 59, 436-442.

[26] Hagmar, J., Brackmann, C., Gustavsson, T., Enejder, A., Image analysis in nonlinear microscopy. J. Opt. Soc. Am. A 2008, 25, 2195-2206.

[27] Abramoff, M. D., Magelhaes, P. J., Ram, S. J., Image processing with ImageJ. Biophotonics International 2004, 11, 36-42.

[28] Bligh, E. G., Dyer, W. J., A rapid method of total lipid extraction and purification. Can. J. Biochem. Physiol. 1959, 37, 911917.

[29] Clausen, M. K., Christiansen, K., Jensen, P. K., Behnke, O., Isolation of lipid particles from baker's yeast. FEBS Lett. 1974, 43, 176-179.

[30] Usaite, R., Jewett, M. C., Oliveira, A. P., Yates, J. R., 3rd et al., Reconstruction of the yeast Snf1 kinase regulatory network reveals its role as a global energy regulator. Mol. Syst. Biol. 2009, 5, 319, 319-330.

[31] Czabany, T., Wagner, A., Zweytick, D., Lohner, K. et al., Structural and biochemical properties of lipid particles from the yeast Saccharomyces cerevisiae. J. Biol. Chem. 2008, 283, 17065-17074.

[32] Pawley, J. B. (Ed.), Handbook of Biological Confocal Microscopy, Springer, New York, 2006.

[33] Modig, T., Liden, G., Taherzadeh, M. J., Inhibition effects of furfural on alcohol dehydrogenase, aldehyde dehydrogenase and pyruvate dehydrogenase. Biochem. J. 2002, 363, 769-776. 